\title{
A Certain Type of Wheeled Self-propelled Gun Independent Suspension Stress Analysis
}

\author{
Liu Xinyun ${ }^{a}$, Ma Jisheng ${ }^{b}$ \\ Artillery Department, Mechanical Engineering College, Heibei Shijiazhuang 050003, China \\ aliu9202111@163.com, bmjs_xxy@163.com
}

Keywords: Wheeled Self-propelled Gun, rigid-flexible coupling, stress and strain

\begin{abstract}
For wheeled self-propelled gun dynamics simulation, the study did not consider of the flexibility of the suspension system currently. And wheeled self-propelled gun with complex road and huge fire load, suspension arm are more likely to happen elastic deformation and fatigue damage compared with civil car. In order to improve the model accuracy and explore the stress-strain law, established a rigid-flexible coupling wheeled self-propelled gun vehicle model based on multi-body dynamics, finite element method, and artillery launch dynamics theory. By analyzing the driving and firing model with different conditions, finally to obtain the position of the suspension system prone to damage, easy to have the damage condition and the maximum stress and strain results. Studies have shown that wheeled self-propelled gun dynamic model establishment of flexible suspension is necessary. The analysis results of self-propelled guns suspension system damage mechanism analysis, the fatigue strength analysis has certain reference value.
\end{abstract}

\section{Introduction}

A certain type of wheeled self-propelled guns is independently developed by the first kind of wheeled self-propelled guns in our country, it uses a $6 \times 6$ drive mode chassis and $100 \mathrm{~mm}$ high chamber pressure smoothbore gun [1]. Assault gun is a kind of used for short distance fire support for infantry armored fighting vehicles. It is a kind of offensive weapons, requires high mobility, the big fire to provide rapid and strong support. This type of weaponry combat mode determines the firing and driving vibration response all after six independent suspension. In the process of actual use, some self-propelled gun suspension arm to work long hours produce damage cracks and fracture failure phenomenon. Therefore, analysis of suspension system failure mechanism and the internal stress and strain regularity is necessary. At present, the study of suspension system has a lot of, such as the consideration of automobile suspension flexible vehicle ride comfort study, or optimization analysis of based on the vehicle suspension rigid-flexible coupling $[2,3]$. But for wheeled self-propelled gun suspension rigid-flexible coupling analysis rarely, most references adopt rigid cylindrical rod are interconnected. So use Solidworks, Adams, Abaqus software to establish the whole gun of rigid-flexible coupled dynamics model includes flexible body and arm. Simulation of its driving and firing process and analysis arm's stress and strain regularity, in order to resolve the question of the utility of the self-propelled gun.

\section{The Entire Vehicle Rigid - Flexible Coupling Model}

Walking Part of the Model. Walk part can be simplified as bodywork, independent suspension, tires, including suspension refers to the general term for all power transmission device between the body and the wheel [4]. Self-propelled gun of the suspension system by the completely same structure of six different length of double wishbone typed independent suspension frame. The structure is divided into A-shaped lower arm, single upper arm, steering knuckle, hydraulic shock absorbers, coil springs. Upper and lower arm connected to the steering knuckle with spherical bearing pair, connected with car body with revolute pair. Because do not consider turning performance, simplify the tie rod for point motion constraints. Coil springs and nonlinear hydraulic dampers is determined by the stiffness and damping characteristic curve [1]. To establish upper and lower arm suspension 
finite element model, it uses a 10-node quadratic tetrahedral element mesh, wherein the upper arm is divided 21955 elements, and lower arm is divided 68,632 elements. The arm meshing as shown in Figure 1.

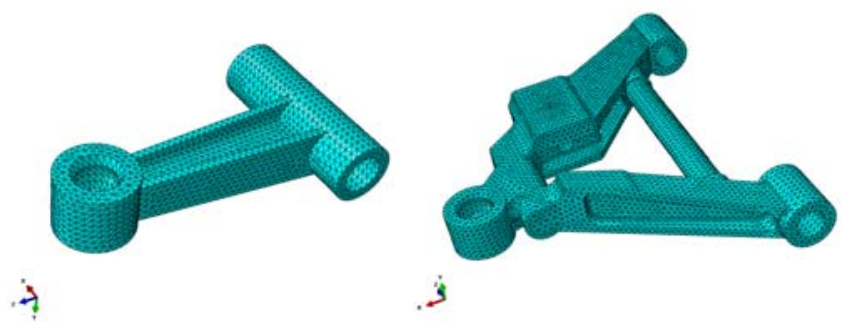

Fig. 1 Suspension upper and lower arm meshing

Modal analysis of the model, take the first 50 modal set of information, generated with the stress and strain modal neutral file (MNF) into Adams.

Fire Part of the Model. In order to more accurately simulate the back seat and the recoil at the time of firing, using modal synthesis method to simplify the body tube model for soft body. Establish Abaqus finite element model of the barrel, divided 45846 eight - node hexahedral elements and modal analysis, take the top 50 order modal is set as the main mode into Adams. Flexible joints breech, muzzle brake, cradle by joints and coupling node. Using Adams function editor, spline curve data, user subroutine to wirte forces of gun bore, recuperator force, friction, recoil mechanism force. The gun loaded directly by the bore pressure curve obtained from the internal ballistics calculation. recuperator force and recoil mechanism force respectively as formula (1) and (2).

$$
P_{f}=P_{f 0}\left(\frac{S_{0}}{S_{0}-X}\right)^{n}
$$

Including: $S_{0}$ recuperator reduced height, $P_{f 0}$ recuperator at the beginning of force, $X$ displacement of the back seat.

$$
\Phi_{0}=\frac{K_{1} r}{2 g}\left[\frac{\left(A_{0}-A_{p}\right)^{3}}{a_{x}^{2}}+\frac{K_{2}}{K_{1}} \frac{A_{t i}^{3}}{\Omega^{2}}\right] V^{2}
$$

Including: $A_{0}$ piston work area, $A_{p}$ control loop hole area, $A_{f j}$ recoil controller work area, $\Omega$ tributary of the minimum cross-sectional area, $a_{x}$ fluid flow hole area, $K_{1} \& K_{2}$ mainstream and tributaries hydraulic resistance coefficient, $r$ liquid heavy, $V$ recoil velocity.

The ground model. Random road excitation is one of the main factors influencing the key position of vibration signal. The road random signal can only use various statistical characteristics to represent it. Available power spectral density to characterize its statistical power spectral characteristics. Using Fourier inverse transformation method of road roughness refactoring. This method is a value obtained by a known power spectral corresponding to a series of discrete Fourier changes. Then normally distributed random sequence by taking the phase information after Fourier transform as the phase angle input, construction of a new frequency-domain signal. Finally, carries on the Fourier inverse transformation to get road roughness random sequence. This method has a rigorous theoretical basis, the resulting road signal has best fitting degree with standard road spectrum. Eventually established as shown in figure 2 the integral rigid-flexible coupling wheeled self-propelled gun dynamics model. 


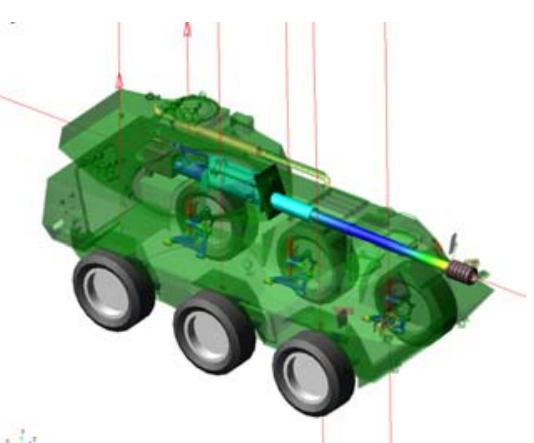

Fig. 2 The integral rigid-flexible coupling gun dynamics model

\section{The Simulation Calculation and Analysis}

Driving Analysis of Random Rough Road. To be able to obtain the advantages and differences in rigid-flexible coupling model by comparing, respectively to the rigid arm suspension and flexible arm suspension self-propelled guns on D class random road driving simulation analysis. With $2 \mathrm{~s}$ acceleration time and $15 \mathrm{~km} / \mathrm{h}$ speed, measuring suspension kingpin inclination angle and the tire camber angle. Kingpin inclination angle is refer to the angle projector in the vehicle transverse plane between the plumb line and the main axis of the pin. When the wheel hop kingpin inclination can improve vehicle steering agility. Tire camber angle is refer to the angle between the wheel tread and the vertical line. Self-propelled gun kingpin inclination angle and tire camber angle change curve with the wheel jumping up and down stroke is shown in figure 3.
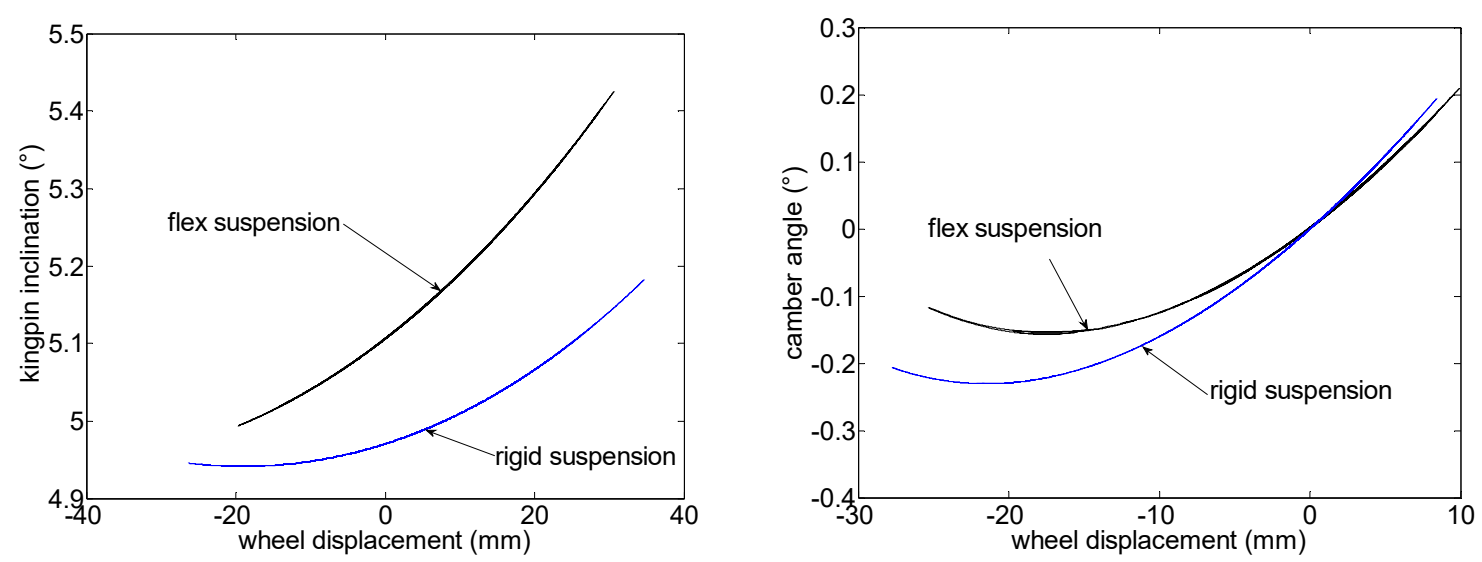

Fig. 3 Kingpin inclination angle and wheel camber angle change

From the results can be seen, there is a big difference between rigid suspension system and flexible suspension system. This also proves the necessity of establishing wheeled self-propelled gun rigid-flexible coupling suspension system. And flexible upper and lower arm can increase the accuracy of the model.

Stationary Firing Analysis. In front of the firing analysis, need to verify this firing model. Model validation is the only way to determine the accuracy of the model. The model is placed on a flat road, and each wheel brake. Barrel at 0 degree angle of site and 0 degrees direction angle, to simulation of self-propelled gun stationary firing process. Extracted the simulation data comparing with the corresponding experimental data, the results are shown in table 1.

Table 1 Model validation

\begin{tabular}{cccc}
\hline & Experimental value & Simulation value & Error \\
\hline Recoil time $(\mathrm{ms})$ & 138.4 & 129.3 & $6.58 \%$ \\
Counter recoil time $(\mathrm{ms})$ & 479.0 & 468.5 & $2.19 \%$ \\
Recoil velocity $\max (\mathrm{m} / \mathrm{s})$ & 10.02 & 10.21 & $1.79 \%$ \\
Recoil displacement $\max (\mathrm{mm})$ & 668.4 & 650.28 & $2.71 \%$ \\
Linear displacement measuring $(\mathrm{mm})$ & 100.5 & 91.0941 & $9.36 \%$ \\
Angular displacement measuring $\left({ }^{\circ}\right)$ & 2.95 & 2.7059 & $8.27 \%$ \\
\hline
\end{tabular}


Comparative experimental and simulation data, errors are allowed within the scope of the project. Shows that the virtual prototype has certain accuracy and reasonableness. Model validation to the next firing simulation calculation result has credibility.

To compare the stress state of the working condition of different firing angle of suspension, take four different direction angle and three different high and low angle of a total of 12 firing angle. Each simulation analysis and extract the suspension maximum stress and strain point. Because of the rear suspension of all working load is the largest, select the rear suspension arm as the research object. The calculation results show that in the selection of 12 firing angle, $60^{\circ}$ direction angle with $20^{\circ}$ high and low angle of maximum stress and strain is the largest, and it is $567.272 \mathrm{Mpa}$ and 0.00196 . While $90^{\circ}$ direction angle with $0{ }^{\circ}$ high and low angle of maximum stress and strain is the least, and it is $451.026 \mathrm{Mpa}$ and 0.00148 . Therefore, it should reduce as far as possible in the actual condition of $60 / 20$ or $30 / 20$ firing angle. The firing angle are more likely to accelerate the suspension arm fatigue damage.

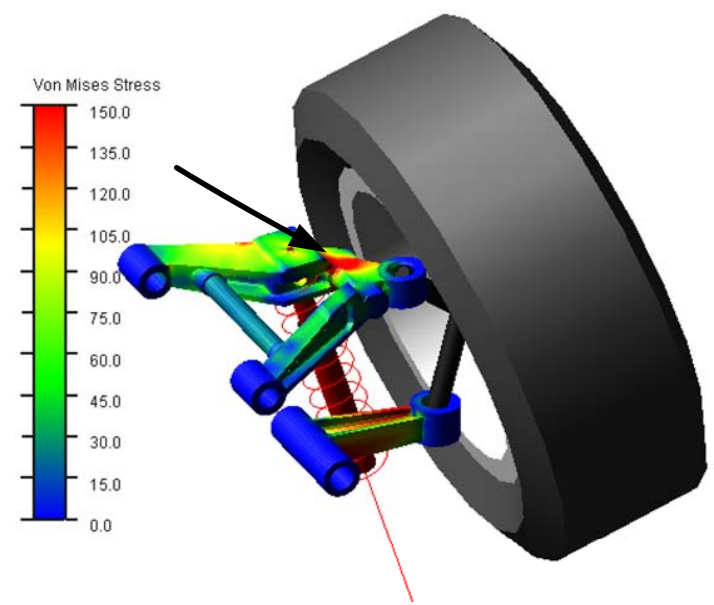

Fig. 4 Suspension arm Mises equivalent stress cloud

Figure 4 is a self-propelled gun after loading forces of gun bore time 0.3945 s rear suspension equivalent stress cloud. This moment lower arm achieves the maximum stress value, places such as the arrow is the largest regional stress. This area is located in the lower arm near the surface of the coil spring connected position. This should be where the suspension system is the most prone to damage failure. In recent years, the actual gun suspension system to produce crack area is consistent, this also proves that the accuracy of the rigid-flexible coupling model. To improve suspension arm structure strength, in this area you can take the position of thick, rounded, replacement materials and other measures.

\section{Summary}

In the first place, for wheeled self-propelled artillery, random road analysis shows that it is necessary to establish contain flexible arm rigid-flexible coupling suspension system. It can more accurately reflect the actual circumstances of the suspension, and it for the future of established dynamic model of wheeled self-propelled guns provides a reference method and theoretical basis. Secondly, the self-propelled gun model to fire simulation in the stationary state, the results show that $60^{\circ}$ direction angle with $20^{\circ}$ high and low angle of maximum stress and strain is the largest, and determining the maximum stress area is located near the bottom surface of the lower arm coil spring position.

\section{References}

[1] Hu Hui-bin, Chen Shu-xiao, Li Lin-lin. Simulation and evaluation on handling stability of wheeled self-propelled gun based on virtual prototype [J]. Journal of System Simulation, 2015, 27(11): 2735-2740. 
[2] Ou Jian, Zhang Qing-lin, Yang E-chuan, Zhang Yong. Rigid-flexible coupling study on ride comfort of car with flexible suspension [J]. Machinery Design \& Manufacture, 2015(02):132-138.

[3] Cai Heng-xing, Jin Zhi-yang, Li Jin-song, Li Mei. Optimization analysis on rigid-flexible coupling mcpherson suspension [J]. Machinery Design \& Manufacture, 2014(09):37-39.

[4] Chen Jia-rui. Construction of Automobile [M]. Beijing:China Communications Press, 2006.

[5] Li Jun, Xing Jun-wen, Qing Wen-jie. ADAMS instance tutorial [M]. Beijing: Beijing institute of technology, 2002. 